\title{
Investigation of the Charge Behavior of PAA Copolymers by Means of Polyelectrolyte Titration
}

\author{
Simona Schwarz ${ }^{1}$, Christine Steinbach¹, Dana Schwarz², Gudrun Petzold ${ }^{1}$, Evgenia Romanova1, \\ Marina Oelmann ${ }^{1}$ \\ ${ }^{1}$ Leibniz-Institut für Polymerforschung Dresden e.V. \\ Hohe Straße 6, 01069, Dresden, Germany \\ simsch@ipfdd.de, steinbach@ipfdd.de, petzold@ipfdd.de, romanova@ipfdd.de, oelmann@ipfdd.de \\ ${ }^{2}$ Charles University in Prague, Faculty of Science, Department of Organic Chemistry \\ Hlavova 2030/8, 128 43, Prague, Czech Republic
}

\section{Extended Abstract}

In this work, the charge density (CD) of polyanions and polycations were investigated by means of polyelectrolyte titration. The $\mathrm{CD}$ is independent on the $\mathrm{pH}$ for strong polyelectrolytes. For week PEL the differences of CD is dependet on the $\mathrm{pH}$. Furthermore, often the $\mathrm{pH}$ is dependent on the concentration of PEL or by the solvent. For all applications it is necessary to determine the CD. Many processes are controlled by the CD. Low, medium, and high charged PEL were examined. Furthermore, the effects of the charge density on the flocculation performance of inorganic kaolin suspensions in the presence of different charged cationic polymers were investigated. The flocculation in the presence of low, medium, and high charge density cationic flocculants (i.e. PC10, PC44, and PC90 respectively) with high molecular weight were compared in terms of the flocculation efficiency and floc sizes.

Keywords: polyelectrolytes, flocculation, solid/liquid separation, Kaolin 